\title{
Changes in diet and physical activity resulting from the Strong Hearts, Healthy Communities randomized cardiovascular disease risk reduction multilevel intervention trial
}

\author{
Sara C. Folta', Lynn Paul ${ }^{2}$, Miriam E. Nelson', David Strogatz ${ }^{3}$, Meredith Graham', Galen D. Eldridge ${ }^{5}$,
} Michael Higgins ${ }^{6}$, David Wing ${ }^{6}$ and Rebecca A. Seguin-Fowler ${ }^{7^{*}}$ (D)

\begin{abstract}
Background: Women living in rural areas face unique challenges in achieving a heart-healthy lifestyle that are related to multiple levels of the social-ecological framework. The purpose of this study was to evaluate changes in diet and physical activity, which are secondary outcomes of a community-based, multilevel cardiovascular disease risk reduction intervention designed for women in rural communities.

Methods: Strong Hearts, Healthy Communities was a six-month, community-randomized trial conducted in 16 rural towns in Montana and New York, USA. Sedentary women aged 40 and older with overweight and obesity were recruited. Intervention participants (eight towns) attended twice weekly exercise and nutrition classes for 24 weeks (48 total). Individual-level components included aerobic exercise, progressive strength training, and healthy eating practices; a civic engagement component was designed to address social and built environment factors to support healthy lifestyles. The control group (eight towns) attended didactic healthy lifestyle classes monthly (six total). Dietary and physical activity data were collected at baseline and post-intervention. Dietary data were collected using automated self-administered 24-h dietary recalls, and physical activity data were collected by accelerometry and self-report. Data were analyzed using multilevel linear regression models with town as a random effect.
\end{abstract}

Results: At baseline, both groups fell short of meeting many recommendations for cardiovascular health. Compared to the control group, the intervention group realized significant improvements in intake of fruit and vegetables combined (difference: 0.6 cup equivalents per day, $95 \% \mathrm{Cl} 0.1$ to $1.1, p=.026$ ) and in vegetables alone (difference: 0.3 cup equivalents per day, $95 \% \mathrm{Cl} 0.1$ to $0.6, p=.016$ ). For physical activity, there were no statistically significant betweengroup differences based on accelerometry. By self-report, the intervention group experienced a greater increase in walking MET minutes per week (difference: 113.5 MET-minutes per week, $95 \% \mathrm{Cl} 12.8$ to $214.2, p=.027$ ).

Conclusions: Between-group differences in dietary and physical activity behaviors measured in this study were minimal. Future studies should consider how to bolster behavioral outcomes in rural settings and may also continue to explore the value of components designed to enact social and environmental change.

Trial registration: clinicaltrials.gov Identifier: NCT02499731. Registered 16 July 2015.

Keywords: Cardiovascular disease, Rural, Community, Nutrition, Diet, Exercise, Physical activity

\footnotetext{
* Correspondence: r.seguin-fowler@ag.tamu.edu

${ }^{7}$ Department of Nutrition and Food Science College of Agriculture and Life

Sciences, Texas A\&M University, College Station, Texas, TX 77843, USA

Full list of author information is available at the end of the article
}

(c) The Author(s). 2019 Open Access This article is distributed under the terms of the Creative Commons Attribution 4.0 International License (http://creativecommons.org/licenses/by/4.0/), which permits unrestricted use, distribution, and 


\section{Background}

Cardiovascular disease (CVD) is the leading cause of death for women in the USA, causing approximately 400,000 female deaths per year [1], and heart disease and stroke are among the leading causes of disability [2]. Annual age-adjusted death rates for heart disease are higher in nonmetropolitan areas compared to metropolitan areas [3]. There is a need to address cardiovascular risk, particularly among rural women, who face unique challenges to accessing healthcare and achieving healthy lifestyle behaviors [4].

There is strong epidemiological evidence for the contribution of diet and physical activity to both cardiovascular health and disease risk among women $[5,6]$. Specific foods, such as fruits and vegetables [7-18], and overall dietary patterns, such as the Dietary Approaches to Stop Hypertension (DASH) $[19,20]$ and Mediterranean $[21,22]$ diets, are associated with reduced risk of CVD. However, few women are meeting recommendations for a heart-healthy diet. A study that used National Health and Nutrition Examination Survey data to assess diet quality among the U.S. population classified $42 \%$ of women as having a poor diet and less than $2 \%$ as having an ideal diet based on the American Heart Association (AHA) 2020 Strategic Impact Goals [23]. The difference between guidelines and intakes may be exacerbated in a rural setting. For example, there is some evidence that adults in rural areas consume fewer fruits and vegetables compared to non-rural counterparts [24].

There is likewise strong evidence for the role of physical activity in prevention of CVD. The AHA score for cardiovascular health includes meeting public health guidelines for physical activity as one of the components [5]. Studies demonstrate that higher amounts or intensities of aerobic activity confer a lower risk of CVD in adults [25]. There is also increasing evidence that resistance training provides additional benefits in reducing CVD risk among women [26]. Sedentary behaviors (sitting, television viewing, screen time, and computer use) have also been examined and appear to be independently associated with increased risk of CVD in adults [27]. Less than one-fifth of women (18\%) are meeting current public health guidelines for aerobic and strengthening physical activity [2], and U.S. adults are spending 6 to 8 $h$ per day engaging in sedentary activities [27]. Adults in rural areas are less likely to meet guidelines for aerobic physical activity [2], although there is some data suggesting that they spend more time in light intensity domestic physical activity compared to urban adults [28].

There are many barriers to consuming a heart-healthy diet and engaging in leisure-time physical activity in rural communities. Low population density typically means fewer supermarkets and fresh food markets. This can result in greater travel time (generally sitting in a vehicle), reduced overall food supply, and diminished quality, quantity, and intake of healthy foods, such fresh fruits and vegetables [29-36]. There is also evidence that access to recreational facilities and fitness classes and activities is limited [37-39] and sidewalks may be lacking [40]. A higher poverty rate [41] can also lead to decreased financial access and purchasing power for both healthier foods [30] and physical activity opportunities [42, 43]. Social and cultural norms and attitudes further challenge achieving a heart-healthy diet and engaging in leisure-time physical activity in rural areas [44-46]. Finally, at a personal level, in rural areas barriers to a healthier diet include knowledge gaps and negative perceptions about nutritious foods, including taste, cost, and preparation time [44, 45, 47, 48]; barriers to physical activity include childcare and caregiving duties, poor health, fear of injury, and lack of motivation [42, 43, 49, 50].

There are few community-based interventions designed for CVD prevention among women in the rural settings $[45,51-55]$. Those that exist utilize behavioral theory, most commonly Social Cognitive Theory and the Transtheoretical Model. However, findings from a systematic review were that primary prevention programs for rural women had little effect on CVD risk factors, especially in the long-term [56]. In recent years, the social-ecological model has gained general endorsement for understanding and changing diet and physical activity behaviors [57-59], and offers promise as an approach that can account for the unique social and environmental barriers in the rural environment. However, only one of the previous studies utilized a socialecological model [53].

The Strong Hearts, Healthy Communities (SHHC) program was designed to address key behavioral targets related to CVD prevention among rural women, including diet and physical activity. The intervention was rooted in the social-ecological model, whereby different components of the program targeted different levels of the model and were informed by Social Cognitive Theory $[60,61]$. For example, at the individual level, the curriculum focused on experiential learning to support participants in developing knowledge, self-efficacy, and skill mastery related to diet and physical activity. At the interpersonal level, out-of-class materials were designed to help participants to engage friends and family in their new activities, thereby encouraging social support. In a civic engagement approach, participants worked together to complete food environment and physical activity assessments and to identify an issue to improve upon in the community (e.g. improving crosswalks, healthy atwork food policy). It was expected that the civic engagement activities would increase both social support and collective efficacy, and empower the women to become 
agents of change for their community, leading to improved food and/or physical activity environments. Civic engagement therefore could help promote built environment and policy changes that further reinforce individual-level change through reciprocal determinism.

In a cluster randomized, controlled trial $S H H C$ led to improvements in weight and body mass index, C-reactive protein, AHA's Life's Simple 7 score, and 10-year risk of cardiovascular disease [62]. This paper expands on these data by examining the secondary outcomes of changes in diet and physical activity resulting from $S H H C$, an intervention designed with the rural context specifically in mind. It is important to understand behavioral outcomes in interventions conducted within a rural context given the specific challenges related to the achievement of a heart-healthy dietary pattern and physical activity in these settings.

\section{Methods}

SHHC was tested in a cluster randomized, controlled trial. The study protocol has been previously published [63]. Randomization occurred at the town level: half of the towns in each state were randomized to the SHHC intervention program $(n=8)$, and half were randomized to a control program $(n=8)$. Towns were matched into pairs by population size, rural-urban community area score, and state, and then the Director of the Cornell Statistical Consulting Unit used JMP software (SAS Institute Inc., Cary, NC, USA) to randomly assign each site in the pair to either the intervention or the control. Study staff enrolled participants.

\section{Recruitment and eligibility}

Towns in Montana (12 towns) and New York (4 towns) were selected by the local lead collaborators (Paul in Montana, Strogatz in New York) in partnership with the Principal Investigator (Seguin-Fowler). Towns needed to meet criteria for rurality based on Rural-Urban Commuting Area [64] and medically underserved areas or population designations [65]. Selected towns also had a county extension educator/agent (Montana) [66] or a health educator affiliated with a local healthcare system (New York) with availability, capacity, and interest in running the program. Extension educators/agents and health educators served as program leaders, rather than research personnel, with program sustainability in mind. In the planning phase of the study, local leaders were involved in community audits and focus group recruitment, and they received extensive training on the program itself. Participants were recruited through flyers, community bulletin boards, social media, radio, direct mail postcards, and newspapers, as well as through churches, healthcare providers, human services, and "word of mouth." Inclusion criteria were female sex, age 40 years or older, overweight or obese (body mass index $\geq 25$ ), sedentary, English-speaking, and had physician's approval to participate. Exclusion criteria were very high resting blood pressure (systolic pressure $>160$ and diastolic pressure $>100$ ), very low or very high resting heart rate $(<60$ or $>100$ beats per minute), or cognitive impairment. The selection process is depicted in Fig. 1. Reach of the $S H H C$ program was calculated as the participation rate: number of enrolled $S H H C$ participants in each town divided by the total number of eligible women as determined U.S. Census data on the percentage of women age 40 and over and Behavioral Risk Factor Surveillance System data on the percentage of overweight/obese adults [67]. Average reach of the SHHC program was $2.6 \%$ [67]. The study was approved by the Cornell University and Bassett Healthcare Network Institutional Review Boards.

\section{Intervention}

The SHHC intervention curriculum was developed based on three evidence-based community programs, two of which target the individual level $[51,68]$ and a third, the HEART Club, which uses an innovative civic engagement approach to catalyze positive social and built environment change [69]. Civic engagement has been hypothesized to increase access to food resources even in rural food deserts [48]. SHHC participants met in groups by town twice per week for hourly sessions for 24 weeks (48 classes) and also attended monthly out-ofclass HEART Club meetings. The intervention occurred from September/October 2015 to March-May in 2016 in Montana and November/December 2015 to June/July 2016 in New York.

The diet component aimed to change dietary patterns for alignment with DASH diet principles [70-73] and the Dietary Guidelines for Americans [74]. The nutrition behavioral aims were to increase fruits and vegetables and encourage mono- and polyunsaturated fats, lean protein, and low- and non-fat dairy; to replace refined grains with whole grains; and to decrease overall calories, desserts, processed foods, sugar-sweetened beverages, saturated fats, and sodium. The physical activities included progressive, moderate-intensity aerobic exercise (typically 20-30 min), such as walking DVDs and aerobic dance in nearly all classes; and progressive strength training (typically $10-20 \mathrm{~min}$; two sets of 10 repetitions) of major muscle groups in about two-thirds of classes, utilizing exercises focusing on both single (bicep curls, chest press) and compound (squats, lunges) muscle groups. Participants were encouraged to increase the intensity of both exercise components throughout the program. Participant materials are available at http://www. strongheartshealthycommunities.org. Feasibility and sustainability in low-resource rural communities were 


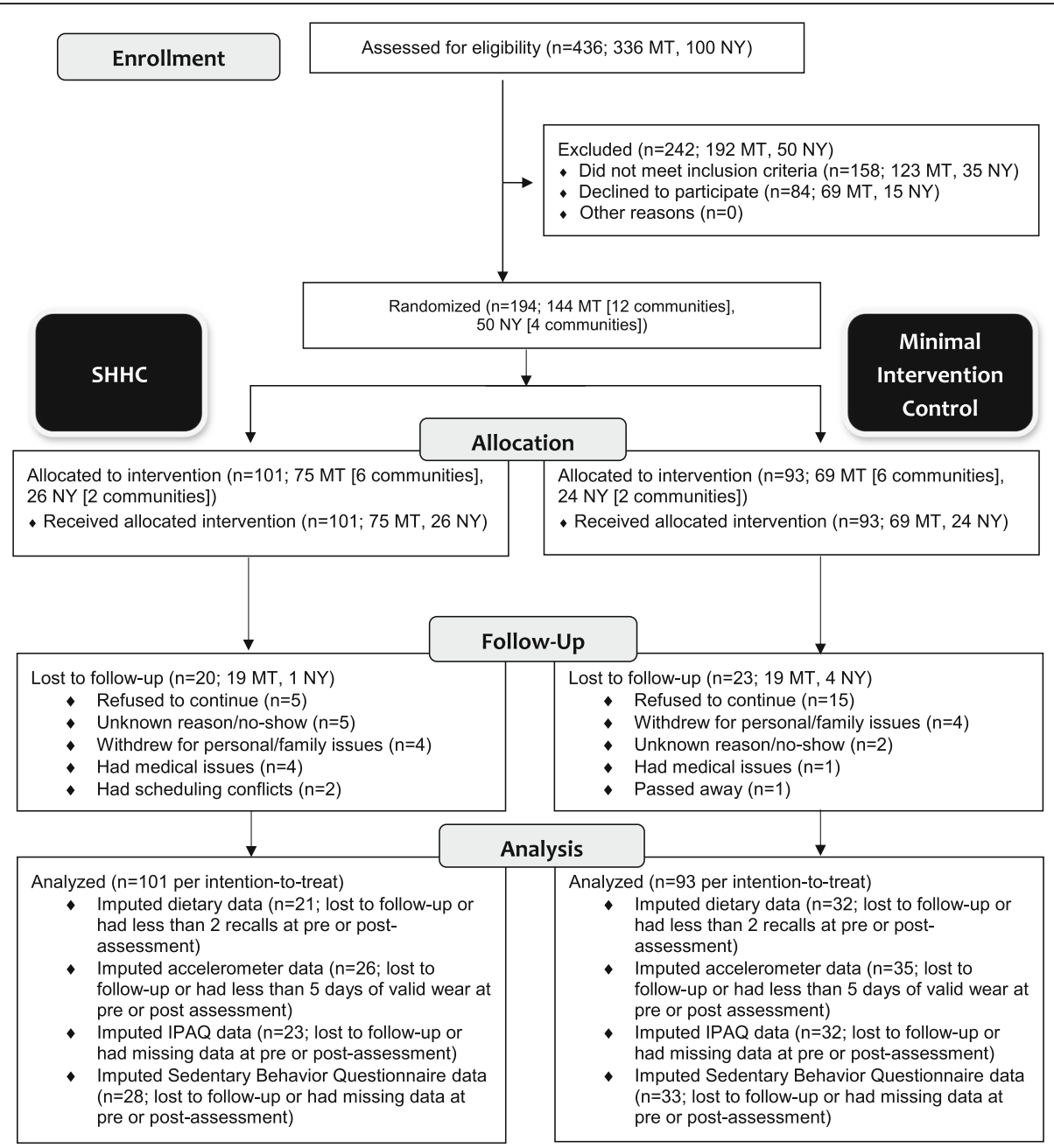

Fig. 1 CONSORT flowchart describing progress of participants through the study. MT, Montana; NY, New York; SHHC, Strong Hearts, Healthy Communities

considered by designing the program so that it could be conducted in community spaces (e.g. church basements, community meeting rooms) and by keeping equipment requirements modest (e.g. yoga mats, hand weights, DVDs).

The control program was designed to serve as a minimal-intervention attention control and was expected to improve knowledge with minimal behavioral impact. Participants in the control program met six times, once per month for an hour, during the six-month intervention period. In this program, a condensed version of $S H H C$ 's curriculum information was presented using a didactic approach with no experiential activity or discussions about civic engagement. Participants did not engage in physical activity during the class sessions.

\section{Measures}

Participants completed a questionnaire that collected basic demographic information at baseline. Demographic questions were derived from national surveys (e.g. U.S. Census). Participants were asked to complete seven dietary recalls during the four-week period just prior to the start of the intervention ("baseline") and again in the four-week period immediately following the 24-week program ("post-intervention"). Participants were asked to wear accelerometers for seven days just prior to the start of the intervention ("baseline") and again for seven days immediately following the conclusion of the program ("post-intervention").

Dietary intake data were collected and analyzed using automated self-administered 24-h dietary recalls (ASA24) [75]. Dietary data were included in analysis if at least two of the seven dietary recalls were completed at each time point to determine usual intakes of foods that are not expected to be episodic [76]. Healthy Eating Index (HEI)-2015 scores were calculated for each participant to determine alignment with the Dietary Guidelines for Americans [77]. Overall scores included 12 components: 
total fruits; whole fruits; total vegetables; dark green and orange vegetables and legumes; total grains; whole grains; milk; meat and beans; oils; saturated fat; sodium; and energy from solid fats, alcohol, and added sugars. These scores were then summed to derive the HEI score, which can range from 0 to 100 .

Our primary measurement of physical activity was obtained using the ActiGraph Model GT3XE accelerometers (ActiGraph LLC, Pensacola, FL). Participants were instructed to wear the device at the hip for seven days and only to remove it when sleeping, bathing, or swimming. Data were recorded at $30 \mathrm{~Hz}$ and analyzed using an epoch length of $60 \mathrm{~s}$. Data were screened using current best practices [78], and non-wear time was identified (and excluded) using a widely-used algorithm developed by Choi et al. [79]. Daily level data were excluded if wear time was less than $10 \mathrm{~h}(600 \mathrm{~min})$ in a day, and participant level data were only included if the participant had five or more valid days of wear (i.e. $\geq 3000$ min across five days with $\geq 600$ min each), or four valid days of wear with at least 750 min per day. Because participants were essentially healthy and without disability, Freedson cut-points were used to determine minutelevel intensity of physical activity [80]. Step counts were also determined. A categorical variable was created from the accelerometer data for both pre- and postintervention: average moderate or vigorous physical activity (MVPA) minutes per day as measured by accelerometer was multiplied by 7 to give average minutes of MVPA per week. If average minutes of MVPA per week was greater than or equal to $150 \mathrm{~min}$ then the participant is meeting the physical activity recommendations [25]. If average minutes of MVPA is less than $150 \mathrm{~min}$ per week then the participant is not meeting physical activity recommendations.

We used self-report as a secondary measure of physical activity to help account for the limitations of accelerometry, such as an inability to capture some types of activities (those involving use of the upper extremities, stationary activities, and swimming), and an inability to distinguish the purpose of the physical activity (work, leisure, transportation) [81]. Self-report measures complement objective measures by accounting for these limitations, although they have their own limitations such as recall bias and an inability to account for shorter durations or lower-intensity activities. The International Physical Activity Questionnaire Short Form (IPAQ-SF) was used pre- and post-intervention to collect self-report of physical activity [82-84]. Metabolic equivalent (MET) minutes per week were compiled according to the IPAQ's Guidelines for Data Processing and Analysis [85]. Self-report of sedentary time was obtained using the Sedentary Behavior Questionnaire [86].

\section{Statistical analysis}

Descriptive statistics for the whole sample and by treatment groups were compiled and tabulated. Comparisons of continuous and categorical variables between the groups at baseline were done using t-tests and chi-square tests, respectively. Since the observations are clustered by town, we conducted multilevel linear regression models where town was treated as a random effect. For each diet and physical activity outcome, an unadjusted model was run with pre-post intervention change as the dependent variable, with treatment as a fixed effect and site as a random effect. Adjusted models, which additionally controlled for baseline values of the outcome, age, marital status, and education, were estimated. Missing data were handled using multiple imputation to minimize bias which could have resulted if complete case analysis were used. The imputation was conducted in SAS (PROC MI). Thirty datasets were imputed and SAS PROC MIANALYZE was then used to combine the model results from within each imputed data set into one summary output. We also used multilevel logistic regression to assess whether treatment was associated with meeting the MVPA recommendation (as measured by accelerometry). Generalized linear mixed effects model (PROC GLIMMIX in SAS) was used with site as a random effect, where meeting the recommendation at outcome was a 1 and not meeting a recommendation at outcome was a 0; education, age, marital status, and baseline meeting of recommendation were included as covariates. All tests were two-sided. We adjusted for multiple testing using the Benjamini-Hochberg approach [87] to avoid risk of an inflated type I error based on the large number of significance tests. We applied the method to physical activity and dietary outcomes together and used a false positive rate of $20 \%$. The adjusted $p$-value for significance based on correcting for both the number of within arm and between arm tests (37 outcomes with three statistical tests each for a total of 111 tests) is $p=.0468$. Analyses were conducted using SAS version 9.4 (SAS Institute Inc., Cary, NC, USA).

\section{Results}

Of a total of 194 study participants, 141 had pre-post 24-h dietary recall data and 133 had pre-post accelerometry data; missing data were imputed (Fig. 1). There were no statistically significant differences in demographic characteristics between the intervention and control group in the analytic sample (Table 1). At baseline, $28 \%$ of participants $(n=54)$ completed seven dietary recalls and $71 \%$ $(n=137)$ completed at least five dietary recalls; $46 \%(n=$ 90) had a full seven days of valid accelerometer wear. There was a statistically significant difference in age at baseline between participants who had completed at least two dietary recalls at post-intervention and those who had not (59.6 years for completers vs. 56.5 for non-completers, 
Table 1 Baseline Characteristics of Participants by Intervention Condition

\begin{tabular}{|c|c|c|c|c|}
\hline Characteristic & $\begin{array}{l}\text { Total } \\
(n=173 \text { in } 16 \\
\text { towns })\end{array}$ & $\begin{array}{l}\text { Control } \\
(n=81 \text { in } 8 \\
\text { towns })\end{array}$ & $\begin{array}{l}\text { SHHC } \\
(n=92 \text { in } 8 \\
\text { towns })\end{array}$ & $\begin{array}{l}\mathrm{p}^{-} \\
\text {value }\end{array}$ \\
\hline Age, mean (SD) & $58.6(9.5)$ & $59.0(96)$ & $58.9(9.5)$ & .98 \\
\hline Income, $\mathrm{n}(\%)^{\mathrm{a}}$ & & & & .24 \\
\hline$<\$ 25,000$ & $33(21)$ & $12(16)$ & $21(26)$ & \\
\hline$\$ 25,000-\$ 50,000$ & $47(30)$ & $26(34)$ & $21(26)$ & \\
\hline$>\$ 50,000$ & $78(49)$ & $39(50)$ & $39(48)$ & \\
\hline Marital status, n (\%) ${ }^{\mathrm{b}}$ & & & & .61 \\
\hline In a relationship (married or member of an unmarried couple) & $122(72)$ & $57(70)$ & $65(74)$ & \\
\hline $\begin{array}{l}\text { Not in a relationship (divorced, widowed, separated, or never been } \\
\text { married) }\end{array}$ & $47(28)$ & $24(30)$ & $23(26)$ & \\
\hline Educational level, $n(\%)^{c}$ & & & & .88 \\
\hline High school or less & $38(23)$ & $17(21)$ & $21(24)$ & \\
\hline Technical or vocational school/some college & $52(31)$ & $24(30)$ & $28(32)$ & \\
\hline College graduate & $52(31)$ & $26(32)$ & $26(30)$ & \\
\hline Postgrad/professional & $26(15)$ & $14(17)$ & $12(14)$ & \\
\hline Racial/ethnic minority, n (\%) ${ }^{c}$ & $9(5)$ & $4(5)$ & $5(6)$ & .84 \\
\hline Employment status, n (\%) ${ }^{d}$ & & & & .10 \\
\hline Employed for wages or self-employed & $120(71)$ & $62(77)$ & $58(65)$ & \\
\hline Not working or retired & $50(29)$ & $19(23)$ & $31(35)$ & \\
\hline Smoking ${ }^{c}$ & $7(13)$ & $3(12)$ & $4(14)$ & .81 \\
\hline Body mass index, mean (SD) & $35.1(6.3)$ & $35.4(6.7)$ & $34.8(6.0)$ & .56 \\
\hline Weight, mean (SD), kg & $93.7(17.7)$ & $95.6(19.0)$ & $92.0(16.1)$ & .19 \\
\hline$\%$ meeting guidelines for physical activity ${ }^{e}$ & 19.7 & 19.3 & 20.0 & .91 \\
\hline
\end{tabular}

${ }^{\mathrm{a}}$ Total $n=158$ (15 missing)

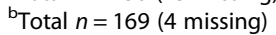

${ }^{\top}$ Total $n=168$ (5 missing)

${ }^{\mathrm{d}}$ Total $n=170$ (3 missing)

${ }^{\mathrm{e}}$ Total $n=162$ (11 missing)

$p=.03)$. There were no statistically significant differences between those who had complete pre-post accelerometry data and those who did not. There were no statistically significant differences in baseline dietary (Table 2) or physical activity (Table 3 ) measures between the intervention and control groups ( $p \geq .05$ in all cases).

For both groups, on average, intakes of sodium, added sugars, fiber, and fruits and vegetables failed to meet recommendations for cardiovascular health $[88,89]$ at baseline (Table 2). The average HEI diet quality score placed participants in both groups slightly below the U.S. national average of 59 [90].

Both groups also fell short of the public health recommendation for physical activity at baseline. While $150 \mathrm{~min}$ per week of at least moderate activity or 75 min per week of vigorous activity is recommended [91], participants obtained closer to $100 \mathrm{~min}$ per week of MVPA, with about half as moderate activity, based on daily averages (Table 3 ). Approximately $20 \%$ of participants were meeting the recommendation and there were no differences between arms at baseline (Table 1). Average daily step counts were approximately half of the widely-promoted recommendation of 10,000 steps per day [92].

Compared to the control group, the intervention group realized statistically significant improvements in intake of fruit and vegetables combined (difference: 0.6 cup equivalents per day, 95\% CI 0.1 to $1.1, p=.026$ ) and in vegetables alone (difference: 0.3 cup equivalents per day, $95 \%$ CI 0.1 to $0.6, p=.016$ ) (Table 2). For physical activity, there were no statistically significant differences between the intervention and control groups based on accelerometry, our primary measure of physical activity (Table 3). By self-report, compared to the control group, the intervention group experienced a greater increase in walking MET-minutes per week (difference: 113.5 MET-minutes per week, 95\% CI 12.8 to $214.2, p=.027)$. No other statistically significant differences in diet or physical activity outcomes between groups were observed. 
Table 2 Dietary Outcomes at Baseline and Post-Intervention by Treatment Group

\begin{tabular}{|c|c|c|c|c|c|c|c|c|c|c|}
\hline \multirow[t]{3}{*}{ Outcome } & \multirow{2}{*}{\multicolumn{2}{|c|}{$\begin{array}{l}\text { Baseline } \\
\text { Mean (SD) }\end{array}$}} & \multirow{2}{*}{\multicolumn{2}{|c|}{$\begin{array}{l}\text { Post-intervention, } \\
\text { Mean (SD) }\end{array}$}} & \multicolumn{6}{|l|}{ Pre-post change } \\
\hline & & & & & \multicolumn{2}{|l|}{ Control } & \multicolumn{2}{|c|}{ SHHC Intervention } & \multicolumn{2}{|l|}{$\begin{array}{l}\text { Adjusted } \\
\text { difference }^{b, c}\end{array}$} \\
\hline & Control & $\begin{array}{l}\text { SHHC } \\
\text { Intervention }\end{array}$ & Control & $\begin{array}{l}\text { SHHC } \\
\text { Intervention }\end{array}$ & $\underset{\mathrm{a}}{\text { Mean }}(95 \% \mathrm{Cl})$ & $\begin{array}{l}\mathrm{p}^{-} \\
\text {value }\end{array}$ & $\underset{\mathrm{a}}{\mathrm{a}} \mathrm{Mean}(95 \% \mathrm{Cl})$ & $\begin{array}{l}p^{-} \\
\text {value }\end{array}$ & $\begin{array}{l}\text { Mean }(95 \% \\
\text { Cl) }\end{array}$ & $\begin{array}{l}\mathrm{p}- \\
\text { value }\end{array}$ \\
\hline Kcal & $\begin{array}{l}1826.4 \\
(59.2)\end{array}$ & $\begin{array}{l}1744.2 \\
(48.9)\end{array}$ & $\begin{array}{l}1560.3 \\
(65.6)\end{array}$ & $\begin{array}{l}1562.8 \\
(54.0)\end{array}$ & $\begin{array}{l}-233.5(- \\
389.0,-78.0)\end{array}$ & .003 & $\begin{array}{l}-183.9(- \\
332.3,-35.4)\end{array}$ & .015 & $\begin{array}{l}49.6(- \\
144.3,243.6)\end{array}$ & .616 \\
\hline Carbohydrate, $g$ & $\begin{array}{l}198.7 \\
(6.9)\end{array}$ & $190.3(6.3)$ & $\begin{array}{l}168.4 \\
(7.9)\end{array}$ & $174.7(7.3)$ & $\begin{array}{l}-26.8(-46.9,- \\
6.8)\end{array}$ & .009 & $\begin{array}{l}-16.4(-36.5 \\
3.8)\end{array}$ & .111 & $\begin{array}{l}10.4(-15.6 \\
36.5)\end{array}$ & .432 \\
\hline Protein, $\mathrm{g}$ & $\begin{array}{l}75.8 \\
(2.2)\end{array}$ & $73.2(1.8)$ & $\begin{array}{l}72.9 \\
(2.8)\end{array}$ & $70.0(2.3)$ & $-1.5(-7.1,4.1)$ & .593 & $-2.7(-7.6,2.3)$ & .289 & $\begin{array}{l}-1.2(-7.7 \\
5.4)\end{array}$ & .729 \\
\hline Fiber, $g$ & $\begin{array}{l}15.6 \\
(0.7)\end{array}$ & $15.2(0.5)$ & $\begin{array}{l}14.5 \\
(0.8)\end{array}$ & $15.9(0.8)$ & $-0.9(-2.9,1.1)$ & .355 & $0.7(-1.2,2.6)$ & .461 & $1.6(-0.8,4.1)$ & .195 \\
\hline Total fat, $g$ & $\begin{array}{l}79.5 \\
(3.2)\end{array}$ & $76.1(2.5)$ & $\begin{array}{l}66.1 \\
(3.6)\end{array}$ & $65.8(2.8)$ & $\begin{array}{l}-12.1(-20.6,- \\
3.6)\end{array}$ & .005 & $\begin{array}{l}-10.5(- \\
18.3,-2.6)\end{array}$ & .009 & $\begin{array}{l}1.7(-8.9 \\
12.2)\end{array}$ & .757 \\
\hline Saturated fat, $g$ & $\begin{array}{l}26.9 \\
(1.2)\end{array}$ & $26.2(1.0)$ & $\begin{array}{l}22.9 \\
(1.3)\end{array}$ & $21.9(1.1)$ & $\begin{array}{l}-3.6(-6.8,- \\
0.5)\end{array}$ & .023 & $\begin{array}{l}-4.4(-7.2,- \\
1.6)\end{array}$ & .004 & $1.4(-2.4,5.1)$ & .731 \\
\hline Monounsaturated fat, $g$ & $\begin{array}{l}28.7 \\
(1.1)\end{array}$ & $27.4(0.9)$ & $\begin{array}{l}22.5 \\
(1.2)\end{array}$ & $23.2(1.0)$ & $\begin{array}{l}-5.8(-8.8,- \\
2.7)\end{array}$ & $<.001$ & $\begin{array}{l}-3.2(-5.8,- \\
0.6)\end{array}$ & .002 & $1.9(-1.6,5.5)$ & .479 \\
\hline Polyunsaturated fat, $\mathrm{g}$ & $\begin{array}{l}17.1 \\
(0.9)\end{array}$ & $16.1(0.6)$ & $\begin{array}{l}15.2 \\
(1.0)\end{array}$ & $15.4(0.7)$ & $-1.5(-3.8,0.9)$ & .233 & $-0.4(-2.5,1.6)$ & .684 & $1.0(-1.8,3.9)$ & .481 \\
\hline Dietary cholesterol, mg & $\begin{array}{l}274.0 \\
(12.4)\end{array}$ & $281.6(14.6)$ & $\begin{array}{l}263.5 \\
(19.7)\end{array}$ & $267.0(14.1)$ & $\begin{array}{l}-10.4(-54.8 \\
33.9)\end{array}$ & .644 & $\begin{array}{l}-9.7(-50.4, \\
31.0)\end{array}$ & .640 & $\begin{array}{l}0.7(-55.0 \\
56.5)\end{array}$ & .979 \\
\hline Sodium, mg & $\begin{array}{l}3146.7 \\
(104.0)\end{array}$ & $\begin{array}{l}3118.3 \\
(89.2)\end{array}$ & $\begin{array}{l}2841.6 \\
(116.3)\end{array}$ & $\begin{array}{l}2775.5 \\
(91.5)\end{array}$ & $\begin{array}{l}-266.4(- \\
505.7,-27.0)\end{array}$ & .029 & $\begin{array}{l}-299.6(- \\
525.1,-74.1)\end{array}$ & .009 & $\begin{array}{l}-33.2(- \\
331.0,264.6)\end{array}$ & .827 \\
\hline HEI 2015 Score & $\begin{array}{l}56.3 \\
(1.3)\end{array}$ & $57.0(1.1)$ & $\begin{array}{l}57.6 \\
(1.6)\end{array}$ & $61.9(1.4)$ & $1.0(-2.8,4.8)$ & .603 & $4.9(1.4,8.4)$ & .006 & $3.9(-1.0,8.8)$ & .119 \\
\hline \multicolumn{11}{|l|}{ Average daily intake of... } \\
\hline $\begin{array}{l}\text { Fruits and vegetables in cup } \\
\text { equivalents }\end{array}$ & $2.6(0.1)$ & $2.6(0.1)$ & $2.2(0.2)$ & $2.8(0.2)$ & $\begin{array}{l}-0.5(-0.8 \\
-0.1)\end{array}$ & .023 & $0.1(-0.2,0.5)$ & .529 & $0.6(0.1,1.1)$ & .026 \\
\hline Fruits in cup equivalents & $0.9(0.1)$ & $1.0(0.1)$ & $0.8(0.1)$ & $1.1(0.1)$ & $-0.1(-0.4,0.2)$ & .348 & $0.1(-0.2,0.4)$ & .401 & $0.3(-0.1,0.6)$ & .179 \\
\hline Vegetables in cup equivalents & $1.7(0.1)$ & $1.6(0.1)$ & $1.4(0.1)$ & $1.7(0.1)$ & $\begin{array}{l}-0.3(-0.5,- \\
0.1)\end{array}$ & .004 & $0.0(-0.2,0.2)$ & .866 & $0.3(0.1,0.6)$ & .016 \\
\hline $\begin{array}{l}\text { Whole grains in oz. } \\
\text { equivalents }\end{array}$ & $0.8(0.1)$ & $0.8(0.1)$ & $1.0(0.1)$ & $0.9(0.1)$ & $0.2(-0.1,0.5)$ & .230 & $0.1(-0.1,0.4)$ & .360 & $\begin{array}{l}-0.1(-0.4, \\
0.3)\end{array}$ & .738 \\
\hline $\begin{array}{l}\text { Refined grains in oz. } \\
\text { equivalents }\end{array}$ & $4.4(0.3)$ & $3.9(0.2)$ & $3.6(0.3)$ & $3.3(0.2)$ & $-0.6(-1.2,0.1)$ & .109 & $-0.6(-1.3,0.0)$ & .058 & $\begin{array}{l}-0.1(-0.9, \\
0.8)\end{array}$ & .871 \\
\hline $\begin{array}{l}\text { Seafood high in n-3 fatty acids } \\
\text { in oz. equivalents }\end{array}$ & $0.0(0.0)$ & $0.1(0.0)$ & $0.2(0.1)$ & $0.2(0.1)$ & $0.1(0.0,0.3)$ & .170 & $0.1(0.0,0.2)$ & .069 & $0.0(-0.2,0.2)$ & .736 \\
\hline Legumes in oz. equivalents & $0.1(0.0)$ & $0.1(0.0)$ & $0.1(0.0)$ & $0.1(0.0)$ & $0.0(0.0,0.0)$ & .926 & $0.0(0.0,0.1)$ & .237 & $0.0(0.0,0.1)$ & .335 \\
\hline Oils in grams ${ }^{d}$ & $\begin{array}{l}22.8 \\
(1.4)\end{array}$ & $19.8(1.0)$ & $\begin{array}{l}19.9 \\
(1.5)\end{array}$ & $19.9(1.3)$ & $-1.7(-5.2,1.8)$ & .333 & $0.0(-3.5,3.4)$ & .981 & $1.7(-3.0,6.4)$ & .481 \\
\hline Solid fats in grams ${ }^{e}$ & $\begin{array}{l}38.9 \\
(1.8)\end{array}$ & $38.9(1.7)$ & $\begin{array}{l}31.5 \\
(2.0)\end{array}$ & $29.7(1.8)$ & $\begin{array}{l}-7.6(-12.7,- \\
2.5)\end{array}$ & .004 & $\begin{array}{l}-9.2(-14.4,- \\
4.1)\end{array}$ & $<.001$ & $\begin{array}{l}-1.7(-8.6, \\
5.2)\end{array}$ & .632 \\
\hline $\begin{array}{l}\text { Added sugars in teaspoon } \\
\text { equivalents }\end{array}$ & $\begin{array}{l}11.9 \\
(0.9)\end{array}$ & $11.9(0.8)$ & $9.9(1.0)$ & $10.1(0.9)$ & $-2.2(-4.8,-0.3)$ & .087 & $-1.9(-4.3,0.5)$ & .123 & $0.3(-3.0,3.7)$ & .847 \\
\hline
\end{tabular}

${ }^{a}$ Significant within-group pre-post changes are bolded and italicized (corrected $p$-value using Benjamini-Hochberg approach is .0468)

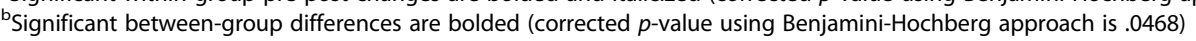

'Adjusted for town, education, age, marital status, and baseline value of the outcome

${ }^{d}$ Fats naturally present in nuts, seeds, seafood; unhydrogenated vegetable oils, except palm oil, palm kernel oil, coconut oils; fat in avocado and olives above allowable amount; $50 \%$ of fat present in stick/tub margarines, margarine spreads (grams)

e Fats naturally present in meat, poultry, eggs, dairy (lard, tallow, butter); hydrogenated/partially hydrogenated oils; shortening, palm, palm kernel, coconut oils; coconut meat, cocoa butter; $50 \%$ of fat in stick/tub margarines, margarine spreads (grams) 
Table 3 Physical Activity Outcomes at Baseline and Post-Intervention by Treatment Group

\begin{tabular}{|c|c|c|c|c|c|c|c|c|c|c|}
\hline \multirow[t]{3}{*}{ Outcome } & \multirow{2}{*}{\multicolumn{2}{|c|}{$\begin{array}{l}\text { Baseline } \\
\text { Mean (SE) }\end{array}$}} & \multirow{2}{*}{\multicolumn{2}{|c|}{$\begin{array}{l}\text { Post-intervention } \\
\text { Mean (SE) }\end{array}$}} & \multicolumn{6}{|l|}{ Pre-post change } \\
\hline & & & & & \multicolumn{2}{|l|}{ Control } & \multicolumn{2}{|c|}{ SHHC Intervention } & \multicolumn{2}{|c|}{ Adjusted difference $^{b, c}$} \\
\hline & Control & $\begin{array}{l}\text { SHHC } \\
\text { Intervention }\end{array}$ & Control & $\begin{array}{l}\text { SHH } \\
\text { Intervention }\end{array}$ & $\begin{array}{l}\text { Mean }(95 \% \mathrm{Cl}) \\
\mathrm{a}\end{array}$ & $\begin{array}{l}p- \\
\text { value }\end{array}$ & $\underset{\mathrm{a}}{\text { Mean }}(95 \% \mathrm{Cl})$ & $\begin{array}{l}p- \\
\text { value }\end{array}$ & Mean $(95 \% \mathrm{Cl})$ & $\begin{array}{l}\mathrm{p}- \\
\text { value }\end{array}$ \\
\hline \multicolumn{11}{|l|}{ Accelerometer } \\
\hline Average daily step count & $\begin{array}{l}4687.9 \\
(243.2)\end{array}$ & $\begin{array}{l}4698.1 \\
(718.2)\end{array}$ & $\begin{array}{l}5220.5 \\
(325.2)\end{array}$ & $\begin{array}{l}5870.5 \\
(304.5)\end{array}$ & $\begin{array}{l}330.5(-626.5 \\
1287.4)\end{array}$ & .498 & $\begin{array}{l}912.4(-498.1 \\
2322.8)\end{array}$ & .201 & $\begin{array}{l}581.9(-976.8 \\
2140.6)\end{array}$ & .462 \\
\hline $\begin{array}{l}\text { Light activity, average } \\
\mathrm{min} / \text { day }\end{array}$ & $\begin{array}{l}305.4 \\
(9.3)\end{array}$ & $307.0(8.8)$ & $\begin{array}{l}313.3 \\
(11.6)\end{array}$ & $328.1(9.9)$ & $\begin{array}{l}4.9(-27.0, \\
36.8)\end{array}$ & .764 & $\begin{array}{l}16.1(-13.9, \\
46.0)\end{array}$ & .292 & $\begin{array}{l}11.2(-29.3 \\
51.6)\end{array}$ & .588 \\
\hline MVPA, average min/day & $14.2(1.4)$ & $14.6(1.5)$ & $17.0(2.5)$ & $22.1(2.6)$ & $2.5(-4.2,9.1)$ & .468 & $7.3(0.9,13.7)$ & .026 & $4.8(-3.5,13.2)$ & .254 \\
\hline $\begin{array}{l}\text { Sedentary time, average } \\
\text { min/day }\end{array}$ & $\begin{array}{l}521.5 \\
(10.2)\end{array}$ & $503.3(9.3)$ & $\begin{array}{l}504.7 \\
(13.0)\end{array}$ & $500.3(11.5)$ & $\begin{array}{l}-13.9(-41.9, \\
14.0)\end{array}$ & .328 & $\begin{array}{l}-6.0(-34.6, \\
22.5)\end{array}$ & .679 & $\begin{array}{l}7.9(-31.2 \\
47.0)\end{array}$ & .690 \\
\hline $\begin{array}{l}\% \text { of activity that is light } \\
\text { intensity }\end{array}$ & $36.2(1.0)$ & $37.0(0.9)$ & $37.4(1.2)$ & $38.6(1.0)$ & $0.9(-2.2,3.9)$ & .569 & $1.3(-1.7,4.3)$ & .388 & $0.4(-3.6,4.4)$ & .831 \\
\hline$\%$ of activity that is MVPA & $1.7(0.2)$ & $1.8(0.2)$ & $2.0(0.3)$ & $2.6(0.3)$ & $0.3(-0.4,1.0)$ & .370 & $0.8(0.1,1.5)$ & .025 & $0.5(-0.4,1.4)$ & .307 \\
\hline $\begin{array}{l}\% \text { of total activity time } \\
\text { that is sedentary }\end{array}$ & $62.1(1.0)$ & $61.2(0.9)$ & $60.6(1.3)$ & $58.9(1.2)$ & $-1.2(-4.3,1.9)$ & .454 & $-2.1(-5.2,1.0)$ & .182 & $-0.9(-5.0,3.2)$ & .655 \\
\hline \multicolumn{11}{|l|}{ IPAQ } \\
\hline Total MET- min/week & $\begin{array}{l}593.8 \\
(98.1)\end{array}$ & $702.0(117.0)$ & $\begin{array}{l}1181.0 \\
(281.0)\end{array}$ & $\begin{array}{l}2080.4 \\
(304.0)\end{array}$ & $\begin{array}{l}499.2(-150.5 \\
1148.8)\end{array}$ & .132 & $\begin{array}{l}1296.6(662.7 \\
1930.5)\end{array}$ & $<.0001$ & $\begin{array}{l}797.4(-92.4 \\
1687.2)\end{array}$ & .079 \\
\hline Walking MET- min/week & $\begin{array}{l}93.0 \\
(18.0)\end{array}$ & $135.0(23.9)$ & $\begin{array}{l}213.8 \\
(31.4)\end{array}$ & $346.1(31.0)$ & $\begin{array}{l}108.2(30.9, \\
185.5)\end{array}$ & .006 & $\begin{array}{l}221.7(141.3, \\
302.2)\end{array}$ & $<.0001$ & $\begin{array}{l}113.5(12.8 \\
214.2)\end{array}$ & .027 \\
\hline Moderate MET-min/week & $\begin{array}{l}197.6 \\
(41.6)\end{array}$ & $277.4(65.8)$ & $\begin{array}{l}432.0 \\
(129.2)\end{array}$ & $\begin{array}{l}548.1 \\
(100.8)\end{array}$ & $\begin{array}{l}168.1(-109.4 \\
445.6)\end{array}$ & .234 & $\begin{array}{l}246.2(8.6, \\
483.9)\end{array}$ & .042 & $\begin{array}{l}78.2(-260.9 \\
417.3)\end{array}$ & .651 \\
\hline Vigorous MET- min/week & $\begin{array}{l}303.2 \\
(73.8)\end{array}$ & $289.5(71.4)$ & $\begin{array}{l}535.1 \\
(187.8)\end{array}$ & $\begin{array}{l}1186.2 \\
(243.4)\end{array}$ & $\begin{array}{l}182.7(-298.2 \\
663.6)\end{array}$ & .456 & $\begin{array}{l}836.1(329.1, \\
1343.1)\end{array}$ & .001 & $\begin{array}{l}653.4(-38.8 \\
1345.6)\end{array}$ & .064 \\
\hline \multicolumn{11}{|c|}{ Sedentary Behavior Questionnaire } \\
\hline $\begin{array}{l}\text { Total hours per week of } \\
\text { sedentary time }\end{array}$ & $75.6(6.5)$ & $76.7(4.8)$ & $81.5(8.6)$ & $79.9(16.7)$ & $\begin{array}{l}2.4(-24.1 \\
28.9)\end{array}$ & .857 & $\begin{array}{l}0.0(-35.2, \\
35.2)\end{array}$ & .998 & $\begin{array}{l}-2.5(-43.5 \\
38.6)\end{array}$ & .905 \\
\hline $\begin{array}{l}\text { Hours per week sitting at } \\
\text { desk }\end{array}$ & $25.5(2.3)$ & $21.2(1.8)$ & $29.2(3.6)$ & $19.7(2.4)$ & $4.4(-3.4,12.1)$ & .270 & $\begin{array}{l}-3.7(-10.5,- \\
3.1)\end{array}$ & .288 & $\begin{array}{l}-8.0(-17.7 \\
-1.6)\end{array}$ & .101 \\
\hline $\begin{array}{l}\text { Hours per week sitting } \\
\text { with friends }\end{array}$ & $10.6(1.7)$ & $10.2(1.2)$ & $11.2(3.4)$ & $13.0(6.3)$ & $0.3(-9.9,10.4)$ & .957 & $1.8(-8.6,12.3)$ & .729 & $1.6(13.3,16.5)$ & .836 \\
\hline $\begin{array}{l}\text { Hours per week sitting } \\
\text { and reading }\end{array}$ & $26.6(2.6)$ & $26.8(2.4)$ & $25.3(2.8)$ & $23.7(2.7)$ & $-1.6(-7.6,4.3)$ & .593 & $\begin{array}{l}-3.5(-10.0 \\
3.1)\end{array}$ & .296 & $\begin{array}{l}-1.8(-10.4 \\
6.7)\end{array}$ & .670 \\
\hline Hours per week driving & $13.0(3.7)$ & $18.5(2.4)$ & $15.7(4.9)$ & $23.6(13.9)$ & $\begin{array}{l}-0.1(-20.6, \\
20.3)\end{array}$ & .990 & $\begin{array}{l}5.5(-24.5 \\
35.6)\end{array}$ & .714 & $\begin{array}{l}5.7(-25.5 \\
36.8)\end{array}$ & .720 \\
\hline
\end{tabular}

${ }^{a}$ Significant within-group pre-post changes are bolded and italicized (corrected p-value using Benjamini-Hochberg approach is .0468)

${ }^{b}$ Significant between-group differences are bolded (corrected p-value using Benjamini-Hochberg approach is .0468)

${ }^{\mathrm{c}}$ Adjusted for town, education, age, marital status, and baseline value of the outcome

\section{Discussion}

This study helps elucidate behavioral outcomes of Strong Hearts, Healthy Communities, one of the first multi-level community-based CVD prevention interventions for rural women. Baseline data from this study confirm the need for interventions to improve behaviors to reduce risk of cardiovascular disease in this population of rural women. Intakes of salt and added sugars were both approximately double AHA recommendations, and participants consumed less fruit, vegetables, and fiber than recommendations. Average minutes of at least moderate physical activity per week were below the recommended 150; at baseline only approximately one-fifth of study participants were meeting these recommendations as measured by accelerometer.

Results suggest minimal between-group behavioral changes in this study. For dietary outcomes, the between-group changes were statistically significant for both fruits and vegetables combined and vegetables alone. These changes reflect a statistically significant within-group decrease in the control group rather than an increase in the $S H H C$ group. For physical activity outcomes, no between-group differences by accelerometry were statistically significant, however there was a statistically significant between-group change in walking MET-minutes per week by self-report. In the primary 
trial report, there were statistically significant betweengroup differences in weight and body mass index and improvement in C-reactive protein [62]. The behavioral data do not correspond well to those findings.

A possible explanation for the discrepancy is that between-group comparisons were diluted by improvements in the control group for several dietary outcomes, including total calories, as revealed by statistically significant within-group results. The minimal-intervention attention control program was designed to provide basic information across a total of six contact hours and implemented based upon community partner preferences and feasibility (versus no program or a delayed program delivery for controls). Both program curricula provided information about CVD rates among women, risk factors, and the basics of a heart-healthy lifestyle including healthy eating and physical activity information. Core educational elements that were common across both programs, particularly around diet, show promise for effectively changing behavior.

For physical activity outcomes, although nonsignificant, between-group differences favored SHHC. It is possible that there was insufficient power to detect changes in these secondary outcomes and therefore an increased probability of a Type II error. For example, per our data for average MVPA, there was an effect size of 0.73 with a standard deviation of 6.4. We therefore would have needed a sample size of 510 individuals to achieve $80 \%$ power at a $p$-value of .05 and accounting for clustering with an intra-class correlation coefficient of .025 . The possibility of a Type II error accounting for the discrepancy between the primary trial report findings and the behavioral outcomes presented here is further supported by findings from this trial indicating that the between-group change in weight was largely accounted for by a change in aerobic fitness, as measured by a step test [93].

Although not our primary measure of physical activity, there were between-group differences in walking METminutes per week by self-report. While both the SHHC and control curricula provided general information about the benefits of physical activity, the SHHC program included the use of walking DVDs in class and emphasized alternative options for out-of-class walking, such as community recreational centers. It is also possible that engagement in HEART Club activities contributed to increased walking. In one study, volunteering, a form of civic engagement, led to increased walking among older women [94].

It is important to consider whether issues with implementation of SHHC could help explain the minimal between-group behavioral results. Although sites were randomized, there could be other factors that influence results that were not accounted for due to the number of sites. However, process evaluation data do not support this possibility. Program leaders had high levels of adherence to the $S H H C$ curriculum (fidelity greater than $80 \%$ ), with exception of only one of the eight intervention sites (68.9\%) [67]. The dose delivered and class effectiveness ratings were also high at all sites except one [67]. Strong implementation of the control curriculum (average 90\% with high levels across all sites, unpublished data) likely contributed to the favorable withingroup dietary changes observed in the control group that may help explain the lack of between-group changes in many of these outcomes.

This study adds to the small body of evidence on behavioral interventions designed specifically for rural women. Heart Smart for Women [54] included behavioral strategies that were similar to $S H H C$; however it was not designed as a multilevel intervention. In that study, there were modest pre-post changes in several outcomes, including fruit consumption and moderate intensity physical activity. A perceived lack of resources, including sources of healthy food, gyms, and safe walking paths, was reported in focus groups conducted with women from the counties where Heart Smart for Women was conducted. In a study conducted in rural counties in upstate New York and Virginia that, like $S H H C$, used a socialecological model, participating women attended a single community-visioning meeting that resulted in a request for community-level changes that were then implemented by a community organization [53]. Community-level changes were modest, however. For example, in New York, physical activity resources were listed on a website. Favorable pre-post changes in fruit and vegetable intake were realized, although these were greater in a group that also included visits by registered nurses to focus on individual-level changes. In this study, HEART Club successes included organizing county-wide health fairs and a restaurant healthy food labeling initiative [62].

Taken together, our results and those of prior studies suggest that while it is possible to achieve good implementation of interventions in the rural environment, attainment of robust behavioral outcomes remains a challenge. The core educational elements from SHHC related to diet and cardiovascular disease provide a basis on which future interventions can build. A multilevel approach was acceptable to participants and they achieved some change in their communities, suggesting that this remains a promising approach. Future studies could continue to explore the value of components designed to enact social and environmental change to better contribute to individual-level behavior change.

This study has several strengths and limitations. It was conducted in multiple rural underserved communities across two states in different regions of the U.S. Thus, findings may generalize to other rural settings. While 
the study population was predominantly white, it reflected the racial/ethnic composition of the rural communities in which the research was conducted. However because of this it is possible the results will not generalize to other populations. The 24-h dietary recall methodology, IPAQ-SF, and Sedentary Behavior Questionnaire have all been validated [84, 86, 95]. Nevertheless, findings are limited by the self-reported nature of these data. Participants were aware of the timeframe during which they would complete the questionnaires and may have made changes in diet and physical activity based on this (reactivity). There were seasonal differences in the pre and post timeframes that likely affected outcomes. For the majority of participants, the baseline period was in September/October, when produce is being harvested and the weather is conducive to outdoor activities, including walking; and post measurements were conducted in March through May, when fresh produce is much less available and the weather is less favorable for outdoor physical activity. This timing may be responsible for the decreases in fruit and vegetable intakes noted in the control group, and may suggest success of the $S H H C$ curriculum in providing women with the behavioral strategies needed to maintain fruit and vegetable intakes despite the seasonal lack of availability.

\section{Conclusions}

Heart disease is an important issue to address in rural communities. There is a need for interventions that address the many barriers to achieving heart-healthy behaviors, particularly because access to healthcare can be extremely limited. The SHHC curriculum achieved success in changing health outcomes [62], however these changes remain largely unexplained in terms of the antecedent diet and physical activity behaviors. Future studies should consider how to bolster behavioral outcomes, possibly by including more and different strategies for affecting multilevel change.

\section{Abbreviations}

AHA: American Heart Association; ASA-24: automated self-administered 24-h dietary recall; Cl: confidence interval; CVD: cardiovascular disease; DASH: Dietary Approaches to Stop Hypertension; HEl: Healthy Eating Index; IPAQ-SF: International Physical Activity Questionnaire Short Form; MET: metabolic equivalents; MVPA: moderate or vigorous physical activity; SHHC: Strong Hearts, Healthy Communities; USA: United States of America

\section{Acknowledgments}

We are grateful for the contribution of the health educators in Montana and New York, Jan Feist, Kristin Pullyblank, and Judy Ward for their programmatic support, and our National Advisory Board members for their contributions to this study.

\section{Authors' contributions}

SCF led the writing of the manuscript and participated in study design and data analysis and interpretation. LP, MEN, and DS participated in study design and interpretation of study results. MG coordinated study implementation, data collection, curriculum development, and data analysis. GDE participated in aspects of data collection and assisted with manuscript preparation. $\mathrm{MH}$ and DW led analysis and interpretation of accelerometry data. RAS conceived of the study and oversaw all aspects of the study design, data analysis and interpretation, and manuscript development. All authors read and approved the final manuscript.

\section{Funding}

This study was supported by grant R01 HL120702 from the National Institutes of Health and the National Heart, Lung, and Blood Institute (NHLBI). Role of the Funders/Sponsors: The funders/sponsors had no role in the design and conduct of the study; collection, management, analysis, and interpretation of the data; preparation, review, or approval of the manuscript; or decision to submit the manuscript for publication.

\section{Availability of data and materials}

The datasets used and analyzed in the current study are available on reasonable request.

\section{Ethics approval and consent to participate}

The study was approved by the Cornell University and Bassett Healthcare Institutional Review Boards. Informed consent was obtained from all study participants.

\section{Consent for publication}

Not applicable.

\section{Competing interests}

The authors have no competing interests to declare.

\section{Author details}

${ }^{1}$ Friedman School of Nutrition, Tufts University, Boston, MA 02111, USA. ${ }^{2}$ College of Education, Health and Human Development, Montana State University, Bozeman, MT 59717, USA. ${ }^{3}$ Center for Rural Community Health, Bassett Research Institute, Cooperstown, NY 13326, USA. ${ }^{4}$ Division of Nutritional Sciences, Cornell University, Ithaca, NY 14853, USA. ${ }^{5}$ Texas A\&M AgriLife Research, College Station, TX 77843, USA. ${ }^{6}$ Exercise and Physical Activity Resource Center, University of California, La Jolla, San Diego, CA 92093-0811, USA. ${ }^{7}$ Department of Nutrition and Food Science College of Agriculture and Life Sciences, Texas A\&M University, College Station, Texas, TX 77843, USA.

Received: 20 September 2018 Accepted: 30 September 2019

Published online: 25 October 2019

\section{References}

1. Murphy SL, Xu J, Kochanek KD, Curtin SC, Arias E. Deaths: final data for 2015. Natl Vital Stat Rep. 2017;66(6):1-75.

2. Benjamin EJ, Virani SS, Callaway CW, Chamberlain AM, Chang AR, Cheng S, et al. Heart disease and stroke Statistics-2018 update: a report from the American Heart Association. Circulation. 2018;137(12):e67-e492.

3. Moy E, Garcia MC, Bastian B, Rossen LM, Ingram DD, Faul M, et al. Leading causes of death in nonmetropolitan and metropolitan areas- United States, 1999-2014. MMWR Surveill Summ. 2017;66(1):1-8.

4. Bennett K, Lopes J, Spencer K, van Hecke S. Rural Women's health: National Rural Health Association Policy Brief: National Rural Health Association; 2013.

5. Lloyd-Jones D, Hong Y, Labarthe D, Mozaffarian D, Appel L, Van Horn L, et al. Defining and setting national goals for cardiovascular health promotion and disease reduction: The American Heart Association's strategic impact goal through 2020 and beyond. Circulation. 2010:121:586-613.

6. Yu E, Rimm E, Qi L, Rexrode K, Albert CM, Sun Q, et al. Diet, lifestyle, biomarkers, genetic factors, and risk of cardiovascular disease in the Nurses' health studies. Am J Public Health. 2016;106(9):1616-23.

7. Alissa EM, Ferns GA. Dietary fruits and vegetables and cardiovascular diseases risk. Crit Rev Food Sci Nutr. 2017:57(9):1950-62.

8. Hartley L, Igbinedion E, Holmes J, Flowers N, Thorogood M, Clarke A, et al. Increased consumption of fruit and vegetables for the primary prevention of cardiovascular diseases. Cochrane Database Syst Rev. 2013;6:CD009874.

9. Wang X, Ouyang Y, Liu J, Zhu M, Zhao G, Bao W, et al. Fruit and vegetable consumption and mortality from all causes, cardiovascular disease, and cancer: systematic review and dose-response meta-analysis of prospective cohort studies. BMJ. 2014;349:g4490. 
10. Aune D, Keum N, Giovannucci E, Fadnes LT, Boffetta P, Greenwood DC, et al. Whole grain consumption and risk of cardiovascular disease, cancer, and all cause and cause specific mortality: systematic review and doseresponse meta-analysis of prospective studies. BMJ. 2016;353:i2716.

11. Flight I, Clifton P. Cereal grains and legumes in the prevention of coronary heart disease and stroke: a review of the literature. Eur J Clin Nutr. 2006. 60(10):1145-59.

12. Mellen PB, Walsh TF, Herrington DM. Whole grain intake and cardiovascular disease: a meta-analysis. Nutr Metab Cardiovasc Dis. 2008;18(4):283-90.

13. Bouchenak M, Lamri-Senhadji M. Nutritional quality of legumes, and their role in cardiometabolic risk prevention: a review. J Med Food. 2013;16(3):185-98.

14. Galli C, Rise P. Fish consumption, omega 3 fatty acids and cardiovascular disease. The science and the clinical trials. Nutr Health. 2009;20(1):11-20.

15. Souza RG, Gomes AC, Naves MM, Mota JF. Nuts and legume seeds for cardiovascular risk reduction: scientific evidence and mechanisms of action. Nutr Rev. 2015;73(6):335-47.

16. Ros E, Hu FB. Consumption of plant seeds and cardiovascular health: epidemiological and clinical trial evidence. Circulation. 2013;128(5):553-65.

17. Hu FB. Plant-based foods and prevention of cardiovascular disease: an overview. Am J Clin Nutr. 2003;78(3 Suppl):544S-51S.

18. Michas G, Micha R, Zampelas A. Dietary fats and cardiovascular disease: putting together the pieces of a complicated puzzle. Atherosclerosis. 2014; 234(2):320-8.

19. Salehi-Abargouei A, Maghsoudi Z, Shirani F, Azadbakht L. Effects of dietary approaches to stop hypertension (DASH)-style diet on fatal or nonfatal cardiovascular diseases--incidence: a systematic review and meta-analysis on observational prospective studies. Nutrition. 2013;29(4):611-8.

20. Siervo M, Lara J, Chowdhury S, Ashor A, Oggioni C, Mathers JC. Effects of the dietary approach to stop hypertension (DASH) diet on cardiovascular risk factors: a systematic review and meta-analysis. Br J Nutr. 2015;113(1):1-15.

21. Rees K, Hartley L, Flowers N, Clarke A, Hooper L, Thorogood M, et al. 'Mediterranean' dietary pattern for the primary prevention of cardiovascular disease. Cochrane Database Syst Rev. 2013;8:CD009825.

22. Martinez-Gonzalez MA, Bes-Rastrollo M. Dietary patterns, Mediterranean diet, and cardiovascular disease. Curr Opin Lipidol. 2014;25(1):20-6.

23. Rehm CD, Penalvo $J$, Afshin A, Mozaffarian D. Dietary intake among US adults, 1999-2012. JAMA. 2016;315(23):2542-53.

24. Lutfiyya MN, Chang LF, Lipsky MS. A cross-sectional study of US rural adults' consumption of fruits and vegetables: do they consume at least five servings daily? BMC Public Health. 2012;12:280

25. Physical Activity Guidelines Advisory Committee. Physical activity guidelines advisory committee report, 2008. Washington, DC: U.S. Department of Health and Human Services; 2008.

26. Drenowatz C, Sui X, Fritz S, Lavie CJ, Beattie PF, Church TS, et al. The association between resistance exercise and cardiovascular disease risk in women. J Sci Med Sport. 2015;18(6):632-6.

27. Endorsed by The Obesity Society, Young DR, Hivert MF, Alhassan S, Camhi SM, Ferguson JF, et al. Sedentary behavior and cardiovascular morbidity and mortality: a science advisory from the American Heart Association. Circulation. 2016:134(13):e262-79.

28. Fan JX, Wen M, Kowaleski-Jones L. Rural-urban differences in objective and subjective measures of physical activity: findings from the National Health and nutrition examination survey (NHANES) 2003-2006. Prev Chronic Dis. 2014;11:E141.

29. Connell CL, Yadrick MK, Simpson P, Gossett J, McGee BB, Bogle ML. Food supply adequacy in the lower Mississippi Delta. J Nutr Educ Behav. 2007; 39(2):77-83.

30. Liese $A D$, Weis $K E$, Pluto $D$, Smith E, Lawson A. Food store types, availability, and cost of foods in a rural environment. J Am Diet Assoc. 2007:107(11):1916-23.

31. Morton L, Blanchard T. Starved for Access: Life in Rural American's Food Desserts. Rural Realities 2007;1(4):https://www.iatp.org/sites/default/files/25 8_2_98043.pdf.

32. Larson N, Story M, Nelson M. Neighborhood environments: disparities in access to healthy foods in the U.S. Am J Prev Med. 2009;36:74-81.

33. Sharkey JR, Johnson CM, Dean WR. Food access and perceptions of the community and household food environment as correlates of fruit and vegetable intake among rural seniors. BMC Geriatr. 2010;10:32.

34. Dean WR, Sharkey JR. Rural and urban differences in the associations between characteristics of the community food environment and fruit and vegetable intake. J Nutr Educ Behav. 2011;43(6):426-33.
35. Jilcott SB, Moore JB, Wall-Bassett ED, Liu H, Saelens BE. Association between travel times and food procurement practices among female supplemental nutrition assistance program participants in eastern North Carolina. J Nutr Educ Behav. 2011:43(5):385-9.

36. Lenardson JD, Hansen AY, Hartley D. Rural and remote food environments and Obesity. Curr Obes Rep. 2015;4(1):46-53.

37. Edwards M, Jilcott S, Moore J, Floyd MF. County level dispartities in access to recreational resources and associations with adult obesity in North Carolina. J Park Recreat Admin. 2011;29(2):39-54.

38. Rivera RL, Dunne J, Maulding MK, Wang Q, Savaiano DA, Nickols-Richardson SM, et al. Exploring the association of urban or rural county status and environmental, nutrition- and lifestyle-related resources with the efficacy of SNAP-Ed (supplemental nutrition assistance program-education) to improve food security. Public Health Nutr. 2018;21(5):957-66.

39. Lo BK, Morgan EH, Folta SC, Graham ML, Paul LC, Nelson ME, et al. Environmental influences on physical activity among rural adults in Montana, United States: views from built environment audits, resident focus groups, and key informant interviews. Int J Environ Res Public Health. 2017:14(10).

40. Robinson JC, Carson TL, Johnson ER, Hardy CM, Shikany JM, Green E, et al. Assessing environmental support for better health: active living opportunity audits in rural communities in the southern United States. Prev Med. 2014;66:28-33.

41. United States Department of Agriculture, Economic Research Service. Rural American At A Glance: 2017 Edition: United States Department of Agriculture; 2017.

42. Parks SE, Housemann RA, Brownson RC. Differential correlates of physical activity in urban and rural adults of various socioeconomic backgrounds in the United States. J Epidemiol Community Health. 2003; 57(1):29-35

43. Olsen J. An integrative review of literature on the determinants of physical activity among rural women. Public Health Nurs. 2013;30(4):288-311.

44. Krummel D, Humphries D, Tessaro I. Focus groups on cardiovascular health in rural women: implications for practice. J Nutr Educ Behav. 2002;34(1):38-46.

45. Tessaro I, Rye S, Parker L, Trangsrud K, Mangone C, McCrone S, et al. Cookin' up health: developing a nutrition intervention for a rural Appalachian population. Health Promot Pract. 2006;7(2):252-7.

46. Sriram U, Morgan EH, Graham ML, Folta SC, Seguin RA. Support and sabotage: a qualitative study of social influences on health behaviors among rural adults. J Rural Health. 2018;34(1):88-97.

47. Folta S, Goldberg J, Lichtenstein A, Seguin R, Reed P, Nelson M. Factors related to cardiovascular risk reduction in midlife and older women: a qualitative study. Prev Chronic Dis 2008;5(1):http://www.cdc.gov/pcd/ issues/2008/jan/06_0156.htm.

48. Smith C, Morton LW. Rural food deserts: low-income perspectives on food access in Minnesota and lowa. J Nutr Educ Behav. 2009:41(3):176-87.

49. Wilcox S, Castro C, King AC, Housemann R, Brownson RC. Determinants of leisure time physical activity in rural compared with urban older and ethnically diverse women in the United States. J Epidemiol Community Health. 2000:54(9):667-72.

50. Seguin R, Connor L, Nelson M, LaCroix A, Eldridge G. Understanding barriers and facilitators to healthy eating and active living in rural communities. J Nutr Metab. 2014;2014:146502.

51. Folta S, Lichtenstein A, Seguin R, Goldberg J, Nelson M. The StrongWomen - healthy hearts program: reducing cardiovascular disease risk factors in rural sedentary, overweight, and obese midlife and older women. Am J Public Health. 2009;99(7):1271-7.

52. Campbell MK, Tessaro I, DeVellis B, Benedict S, Kelsey K, Belton L, et al. Effects of a tailored health promotion program for female blue-collar workers: health works for women. Prev Med. 2002;34(3):313-23.

53. Fahs P, Pribulick M, Canty Williams I, James G, Rovynak V, Seibold-Simpson S. Promoting heart health in rural women. J Rural Health. 2013;29(3):248-57.

54. Khare MM, Koch A, Zimmermann K, Moehring PA, Geller SE. Heart smart for women: a community-based lifestyle change intervention to reduce cardiovascular risk in rural women. J Rural Health. 2014;30(4):359-68.

55. Perry C, Rosenfeld A, Bennett J, Potempa K. Heart-to-heart: promoting walking in rural women through motivational interviewing and group support. J Cardiovasc Nurs. 2007;22(4):304-12.

56. Crouch $\mathrm{R}$, Wilson A, Newbury J. A systematic review of the effectiveness of primary health education or intervention programs in improving rural women's knowledge of heart disease risk factors and changing lifestyle behaviours. Int J Evid Based Healthc. 2011;9(3):236-45. 
57. Sallis J, Cervero R, Ascher W, Henderson K, Kraft M, Kerr J. An ecological approach to creating active living communities. Annu Rev Public Health 2006;27:297-322

58. Story M, Kaphingst KM, Robinson-O'Brien R, Glanz K. Creating healthy food and eating environments: policy and environmental approaches. Annu Rev Public Health. 2008:29:253-72.

59. Glanz K, Rimer B, Viswanath K. Health behavior: theory, research, and practice. San Francisco: Jossey-Bass; 2015.

60. Bandura A. Social foundations of thought and action: a social cognitive theory. Prentice Hall: Englewood Cliffs, NJ; 1986.

61. Kelder S, Hoelscher D, Perry C. How individuals, environments, and health behaviors interact: social cognitive theory. In: Glanz K, Rimer B, Viswanath K, editors. Health behavior: theory, research, and practice. 5th ed. San Francisco: Jossey-Bass; 2015.

62. Seguin RA, Paul L, Folta SC, Nelson ME, Strogatz D, Graham ML, et al. Strong hearts, healthy communities: a community-based randomized trial for rural women. Obesity (Silver Spring). 2018;26(5):845-53.

63. Seguin RA, Eldridge G, Graham ML, Folta SC, Nelson ME, Strogatz D. Strong hearts, healthy communities: a rural community-based cardiovascular disease prevention program. BMC Public Health. 2016;16(1):86.

64. United States Department of Agriculture, Economic Research Service. RuralUrban Continuum Codes. https://www.ers.usda.gov/data-products/ruralurban-continuum-codes/. Accessed 15 June 2019.

65. U.S. Health Resources and Services Administration. Find Shortage Areas: HPSA \& MUA/P by Address. https://www.healthdata.gov/dataset/findshortage-areas-hpsa-muap-address. Accessed 15 June 2019.

66. United States Department of Agriculture, National Institute of Food and Agriculture. https://nifa.usda.gov/extension. Access 15 June 2019.

67. Sriram U, Sandreuter K, Graham M, Folta S, Pullyblank K, Paul L, et al. Process evaluation of strong hearts, healthy communities: a rural community-based cardiovascular disease prevention program. J Nutr Educ Behav. 2019;51(2):138-49.

68. Seguin R, Heidkamp-Young E, Kuder J, Nelson M. Improved physical fitness among older female participants in a nationally disseminated, communitybased exercise program. Health Educ Behav. 2012;39(2):183-90.

69. Seguin $R$, Heidkamp-Young $E$, Juno B, Knott A, Fenton M, Folta $S$, et al. A community-based participatory Reseach pilot initiative to catalyze Postive change in local food and physical activity environments. Austin, TX: International Society for Behavioral Nutrition and Physical Activity Annual Meeting; 2012

70. Appel L, Moore T, Obarzanek E, Vollmer W, Svetkey L, Sacks F, et al. A clinical trial of the effects of dietary patterns on blood pressure. N Engl J Med. 1997;336(16):1117-24.

71. Obarzanek E, Sacks FM, Vollmer WM, Bray GA, Miller ER 3rd, Lin PH, et al. Effects on blood lipids of a blood pressure-lowering diet: the dietary approaches to stop hypertension (DASH) trial. Am J Clin Nutr. 2001;74(1):80-9.

72. Sacks FM, Svetkey LP, Vollmer WM, Appel LJ, Bray GA, Harsha D, et al. Effects on blood pressure of reduced dietary sodium and the dietary approaches to stop hypertension (DASH) diet. DASH-sodium collaborative research group. N Engl J Med. 2001;344(1):3-10.

73. Fung TT, Chiuve SE, McCullough ML, Rexrode KM, Logroscino G, Hu FB. Adherence to a DASH-style diet and risk of coronary heart disease and stroke in women. Arch Intern Med. 2008;168(7):713-20.

74. U.S. Department of Agriculture and U.S. Department of Health and Human Services. Dietary Guidelines for Americans. Washington. DC: U.S. Government Printing Office; 2010. p. 2010.

75. Kirkpatrick SI, Subar AF, Douglass D, Zimmerman TP, Thompson FE, Kahle $L L$, et al. Performance of the automated self-administered 24-hour recall relative to a measure of true intakes and to an interviewer-administered 24h recall. Am J Clin Nutr. 2014;100(1):233-40.

76. National Institutes of Health, National Cancer Institute. Dietary Assessment Primer. https://dietassessmentprimer.cancer.gov/. Access 15 June 2019.

77. Guenther PM, Kirkpatrick SI, Reedy J, Krebs-Smith SM, Buckman DW, Dodd KW, et al. The healthy eating Index-2010 is a valid and reliable measure of diet quality according to the 2010 dietary guidelines for Americans. J Nutr. 2014;144(3):399-407.

78. Matthews CE, Hagstromer M, Pober DM, Bowles HR. Best practices for using physical activity monitors in population-based research. Med Sci Sports Exerc. 2012;44(1 Suppl 1):S68-76.
79. Choi L, Ward SC, Schnelle JF, Buchowski MS. Assessment of wear/nonwear time classification algorithms for triaxial accelerometer. Med Sci Sports Exerc. 2012:44(10):2009-16.

80. Freedson PS, Melanson E, Sirard J. Calibration of the computer science and applications. Inc accelerometer Med Sci Sports Exerc. 1998;30(5):777-81.

81. Prince SA, Adamo KB, Hamel ME, Hardt J, Connor Gorber S, Tremblay M. A comparison of direct versus self-report measures for assessing physical activity in adults: a systematic review. Int J Behav Nutr Phys Act. 2008;5:56.

82. Sallis J, Haskell W, Wood P, Fortmann S, Rogers T, Blaire S, et al. Physical activity assessment methodology in the Five-City project. Am J Epidemiology. 1985:121(1):91-106.

83. Blair S, Haskell W, Ho P, Paffenbarger R, Vranizan K, Farquhar J, et al. Assessment of habitual physical activity by a seven day recall in a community survey and controlled experiments. Am J Epidemiology. 1985; 122(5):794-804.

84. Craig C, Marshall A, Sjostrom M, Bauman A, Booth M, Ainsworth B, et al. International physical activity questionnaire: 12-country reliability and validity. Med Sci Sports Exerc. 2003;35(8):1381-95.

85. International Physical Activity Questionnaire. Guidelines for Data Processing and Analysis of the International Physical Activity Questionnaire (IPAQ). http://www.ipaq.ki.se.

86. Rosenberg D, Norman G, Wagner N, Patrick K, Calfas K, Sallis J. Reliability and validity of the sedentary behavior questionnaire (SBQ) for adults. J Phys Act Health. 2010;7(6):697-705.

87. Glickman ME, Rao SR, Schultz MR. False discovery rate control is a recommended alternative to Bonferroni-type adjustments in health studies. J Clin Epidemiol. 2014;67(8):850-7.

88. Whelton PK, Appel L, Sacco RL, Anderson CA, Antman EM, Campbell N, et al. Sodium, blood pressure, and cardiovascular disease: further evidence supporting the American Heart Association sodium reduction recommendations. Circulation. 2012:126(24):2880-9.

89. Johnson RK, Appel L, Brands M, Howard BV, Lefevre M, Lustig RH, et al. Dietary sugars intake and cardiovascular health: a scientific statement from the American Heart Association. Circulation. 2009;120(11):1011-20.

90. United States Department of Agriculture Center for Nutrition Policy and Promotion. HEl Scores for Americans. https://www.fns.usda.gov/hei-scoresamericans. Accessed 15 June 2019.

91. U.S. Department of Health and Human Services. 2008 Physical Activity Guidelines for Americans. Washington, DC; 2008.

92. Wattanapisit A, Thanamee S. Evidence behind 10,000 steps walking. J Health Res. 2017;31(3):241-8.

93. Pullyblank K, Strogatz D, Folta SC, Paul L, Nelson ME, Graham M, et al. Effects of the Strong Hearts, Healthy Communities intervention on functional fitness of rural women. J Rural Health 2019.

94. Varma VR, Tan EJ, Gross AL, Harris G, Romani W, Fried LP, et al. Effect of community volunteering on physical activity: a randomized controlled trial. Am J Prev Med. 2016;50(1):106-10.

95. Thompson F, Subar A. Dietary assessment methodology. In: Coulston A Boushey C, editors. Nutrition in the. Prevention and Treatment of Disease: Academic Press: 2008

\section{Publisher's Note}

Springer Nature remains neutral with regard to jurisdictional claims in published maps and institutional affiliations.

Ready to submit your research? Choose BMC and benefit from:

- fast, convenient online submission

- thorough peer review by experienced researchers in your field

- rapid publication on acceptance

- support for research data, including large and complex data types

- gold Open Access which fosters wider collaboration and increased citations

- maximum visibility for your research: over $100 \mathrm{M}$ website views per year

At BMC, research is always in progress.

Learn more biomedcentral.com/submissions 